\title{
LA FUCINA DELLA SOVRANITÀ NAZIONALE: LE ELEZIONI NEI SISTEMI LIBERALI ITALIANO E SPAGNOLO
}

\author{
GABRIELE RANZATO
}

In primo luogo debbo avvertire che la comparazione che cercherò di fare del funzionamento dei sistemi elettorali italiano e spagnolo nel cinquantennio che sta a cavallo tra i secoli XIX e XX, risulterà inevitabilmente schematica. Inevitabilmente non solo perchè i limiti di tempo impongono una sinteticità che comporta necessariamente schematismo, ma soprattutto perchè i nostri paesi sono cosí variegati al loro interno, che è impossibile descrivere qualsiasi fenomeno che li riguarda su scala nazionale, senza pagare un tributo allo schematismo.

\section{IL QUADRO ISTITUZIONALE}

Fatta questa avvertenza, che spero possa mettermi al riparo da obiezioni che traggano spunto da esempi relativi ad aree troppo circoscritte, credo sia opportuno premettere alla comparazione dei sistemi elettorali, alcune considerazioni sul complessivo quadro istituzionale entro il quale quelli si iscrivevano. E la considerazione di fondo è che il quadro istituzionale spagnolo presentava delle indubbie maggiori aperture in senso liberale rispetto a quello italiano, a partire dallo stesso ordinamento costituzionale.

Basti considerare già l'origine dei due testi costituzionali, dei quali, l'italiano, è una vera e propria charte octroyée, mentre quello spagnolo è prodotto da Cortes costituenti, anche se bisogna ricordare che a queste di fatto fu imposta la autoinibizione a discutere gli articoli riguardanti il sovrano. Inoltre, per quanto riguarda quest'ultimo, benchè entrambe le costituzioni gli conferissero ampissimi poteri, in quella italiana il re non conosce praticamente limitazioni se non quelle impostegli dalle leggi da egli stesso sanzionate, mentre queIla spagnola prevedeva esplicitamente, nel suo articolo 48 , che «Ningún mandato del rey puede llevarse a efecto si no está refrendado por un ministro».

II prepotere del sovrano nel sistema italiano si evidenzia in particolare nell'ordinamento della giustizia, che, secondo quanto recita l'art. 68 dello Statuto, «emana dal re ed è amministrata in suo nome dai giudici che egli istituisce»; da cui derivò, attraverso la legge del 1859, una forte dipendenza dei giudici dall'esecutivo, più accentuata, almeno nel testo legale, di quella cui era soggetta la magistratura spagnola. E ancor più risalta la supremazia regia sanzionata dallo Statuto nella formazione di uno dei due corpi colegislatori, 
il Senato, che è integralmente nominato dal sovrano, mentre nell'ordinamento spagnolo è per metà di origine elettiva.

In realtà, il carattere estremamente moderato della costituzione italiana le derivava, da una parte, dal luogo e data di nascita -il regno di Sardegna nel 1848, nel corso della prima sfortunata tappa liberal-risorgimentale-; dall'altra, dalla scelta del gruppo dirigente risorgimentale piemontese di stabilire una sostanziale continuità tra regno di Sardegna e regno d'Italia, che implicó il trapasso puro e semplice di ordinamenti e istituizioni dell'uno nell'altro, al punto che la stessa numerazione delle legislature del nuovo Stato unitario non fece che proseguire quella dello Stato sardo-piemontese.

Quella scelta, ispirata dai timori che incuteva ai moderati un processo costituente, non significa però che quel gruppo dirigente si identificasse con la lettera dello Statuto. Cosicchè il sistema politico italiano si convertì molto rapidamente da regime costituzionale a regime parlamentare, il cui asse era di fatto la fiducia parlamentare da cui dipendeva la stabilità dei governi. Ciò non significa che lo Statuto divenne lettera morta, ma che la somma dei poteri che esso prevedeva per il sovrano si trasferi in gran parte sul vero titolare dell'esecutivo cioè il governo. Di qui il carattere notevolmente autoritario e accentrato del sistema liberale italiano, che si evidenzia in particolare nell'ordinamento delle amministrazioni locali, segnato, rispetto a quello spagnolo, da una più limitata partecipazione cittadina - vista la minore ampiezza dell'elettorato amministrativo - da una maggiore ingerenza dell'esecutivo nella formazione degli organi dell'amministrazione - visto che i sindaci erano tutti di nomina governativa, salvo che nei capoluoghi di provincia, e a partire dal 1888-, e da un regime di tutela più severo e invadente.

\section{I sistemi elettorali}

Questa primazia del governo, che si va realizzando parallelamente anche in Spagna - anche se in minor misura, vista la notevole importanza che continua ad avere il re nella pratica costituzionale spagnola, di cui sono tra l'altro testimonianza le cosiddette crisi «orientali» (per gli amici italiani spiego che le crisi orientali erano quelle che avevano origine nel Palacio de Oriente, residenza del re)-; questa primazia del governo, dicevo, e la dipendenza di esso dalla fiducia parlamentare, facevano delle elezioni attraverso cui si formava la Camera uno strumento fondamentale per l'attribuzione di un vasto potere, che fuori della Camera incontrava poche limitazioni. Di qui l'estrema importanza del sistema elettorale e del suo pratico funzionamento.

Ora non vorrei soffermarmi su una comparazione minuta dei sistemi elettorali, le cui differenze ho cercato peraltro di sintetizzare nella tavola I, perchè ritengo che ciò che è importante confrontare è la pratica elettorale e non 
i testi legislativi. Ma vorrei far notare come anche qui risalta la maggior apertura liberale, e anzi liberaldemocratica, dell'ordinamento spagnolo, in cui si introduce il suffragio universale maschile nel 1890 , con più di un ventennio di anticipo rispetto all'Italia, dove viene istituito nel 1912. Anzi, mentre nel caso spagnolo si trattava di un suffragio veramente universale (ancorchè limitato all'universo dei maschi), in quello italiano erano ancora esclusi dal voto gli analfabeti minori di trent'anni. Esclusione che in quel tempo non era di poco rilievo.

Inoltre, anche per quanto riguarda il perio do del suffragio censitario l'ordinamento italiano si presenta più restrittivo di quello spagnolo, giacchè, tra le elezioni del 1861 e quelle del 1880 l'elettorato italiano oscilla tra l'1,9 e il $2,2 \%$ dell'intera popolazione, mentre in Spagna, nel breve periodo di suffragio censitario posteriore alla Restaurazione, tra le elezioni del 1879 e quelle del 1886 , l'elettorato oscilla tra il 4,5 e il $5,7 \%$ dalla popolazione. E questo deslivello tra Italia e Spagna non era dovuto, ovviamente, a una maggiore ricchezza dell'elettorato spagnolo, ma ai più elevati requisiti censitari richiesti della legge elettorale italiana.

Con la riforma elettorale del 1882 , che accanto al censo concede il diritto di voto all'istruzione - se ne richiedeva un livello equivalente alla seconda elementare - in Italia la percentuale dell'elettorato sulla popolazione evidentemente cresce, fino ad arrivare al 9,4\% nel 1892 ('anno precedente con il suffragio universale in Spagna l'elettorato era passato al $27 \%$ della popolazione), ma dopo una drastica revisione delle liste promossa da Crispi, anche in funzione antisocialista, tale percentuale si abbassava al $6,7 \%$ e fino al suffragio universale non avrebbe più raggiunto la quota del 1892.

\section{La pratica elettorale in Italia}

Fin qui per quanto riguarda le istituzioni e i sistemi elettorali. Ma ciò che più ci interessa confrontare è, come dicevo, la realtà del loro funzionamento pratico. E sotto questo profilo gli amici spagnoli sanno meglio di me quanto distante fosse in Spagna quella pratica dal modello liberaldemocratico. In particolare, per quanto riguarda le elezioni, l'adulterazione del voto era un fatto cosí massiccio e sistematico, strumento essenziale come era della perversa regola del turno, da non far apparire esagerata una definizione del sistema spagnolo come sistema pseudorappresentativo.

Non voglio tuttavia addentrarmi ora in un'analisi delle diverse forme in cui si attuava in Spagna lo snaturamento (desvirtuación) del sistema elettorale, perchè in larga misura non potrei dire che cose molto note. Riversandomi di tornarci sopra per mettere a fuoco alcune caratteristiche di quel processo di adulterazione, vorrei piuttosto cominciare con l'occuparmi dell'Italia. E a 
questo proposito la prima cosa da dire è che se ci atteniamo alle testimonianze e alle denunce dell'epoca, che posteriormente hanno trovato espressione nel giudizio della storiografia, il caso dell'Italia non appare molto dissimile da quello spagnolo.

Scrive, ad esempio, Ernesto Ragionieri nella Storia d'Italia Einaudi a proposito delle elezioni del 1892, che «Giolitti fece le elezioni politiche come nessun altro precedente governo italiano, accentuando e perfezionando tutti gli strumenti di intervento e di pressione precedentemente sperimentati» (1). $\mathrm{E}$ in seguito, riferendosi alle elezioni del 1913, le prime a suffragio universale, presiedute anche queste da Giolitti, scrive: «I metodi tradizionali di corruzione e di pressione degli organi periferici dello Stato cedettero il passo, particolarmente nell'Italia meridionale, ma un pò in tutte le zone agrarie più arretrate, ad una massiccia campagna di intimidazione e di violenza, in cui l'apparato dello Stato si combinò con l'intervento attivo dei mazzieri e della mafia» (2).

Ciò che è importante rilevare è pertanto che lungi dall'essere stati introdotti da Giolitti «i metodi di corruzione e di pressione degli organi periferici dello Stato» erano, come si esprime Ragionieri, «tradizionali», e che lo statista piemontese non aveva fatto altro che accentuare e perfezionare «tutti gli strumenti di intervento e di pressione precedentemente sperimentati».

Ed infatti se si scorrono le pagine di Governo e governati in Italia, il noto libro di Pasquale Turiello, apparso nel 1882, durante il periodo del trasformismo inaugurato da Agostino Depretis, si possono leggere molti brani eloquenti come questi: «Nelle elezioni politiche (...), massime dove i collegi sono divisi in frazioni poco sorvegliate e remote, in più di una frazione la maggioranza sopprime la minoranza non solo, ma fa votar talvolta assenti od infermi (...) Nella città di Napoli l'inconveniente diventa più grave (...) I morti e non radiati son molti, le liste son compilate, secondo la legge improvvida che abbiamo, da autorità o da clientele naturalmente partigiane (...) Oltre i brogli, aiutarono ed aiutano in più d'un caso, a corrompere le elezioni, le pressioni governative» (3).

Due anni dopo, Gaetano Mosca il teorico della classe politica, scriveva: «Che i prefetti siano tutti degli agenti elettorali del Ministero è una verità ormai cosí nota che qualunque dimostrazione di essa ci appare superflua", e, come esempio dell'estrema gravità cui poteva giungere l'azione prefettizia, aggiungeva: «n certe province, nel sud specialmente, dove la camorra e la mafia tuttora dispongono di una grande influenza, è sicuro che il governo od i suoi

(1) Storia d'Italia Einaudi, vol. 4, t. III, p. 1.800.

(2) Ivi, p. 1958.

(3) P. Turiello, Governo e governati in Italia 1980 (1. ${ }^{\circ}$ ediz. 1882), pp. 144-46. 
agenti si avvalgono, qualche volta, dell'opera loro nelle elezioni, e che la ricompensano con la concessione di una semiimpunità. In Sicilia (...) nella provincia di $X$ il prefetto $Y$ lascia in carica molti sindaci e non scioglie molti consigli comunali di piccoli comuni, notoriamente concussionari, a patto che diventino suoi fidi agenti elettorali. Nelle ultime elezioni dell'ottobre ottantadue in molti comuni furono prosciolti tutti gli ammoniti e furono dati permessi d'arme a molti notissimi facinorosi per assicurare la riuscita dei candidati ministerialì (4).

Passando al periodo giolittiano, sono note le aspre denunce della corruzione elettorale da parte di Gaetano Salvemini, il quale nel celebre pamphlet dedicato a Giolitti, dal significativo titolo /l ministro delle malavita scriveva: «L'on. Giolitti non è certo il primo uomo di governo dell'Italia una, che abbia considerato il Mezzogiorno come terra di conquista (...) Ma nessuno è stato mai cosí brutale, cosí cinico, cosí spregiudicato come lui (...); nessuno ha fatto un uso più sistematico e più sfacciato, nelle elezioni del Mezzogiorno, di ogni sorta di violenza e di reati» (5). E l'on. Ettore Ciccotti, nel corso della discussione parlamentare relativa alla famosa elezione contestata di Gioia del Colle, che aveva spinto Salvemini a scrivere il suo pamphlet, pronunciava nel suo discorso una frase lapidaria: «Qui in Italia, il deputato, spesse volte, non è eletto dagli elettori: è eletto dal Governo» (6).

Tanto Salvemini che Ciccotti erano socialisti, ma indicazioni del fatto che la realtà non fosse molto diversa da quella che essi denunciavano ci vengono, per cosí dire, dall'interno delle stesse istituzioni. Fra i molti esempi mi sembrano particolarmente significative alcune note autobiografiche dell'ex prefetto Enrico Flores, il quale vi scriveva: «Nel 1897, in occasione delle elezioni politiche (...) fui inviato come Commissario regio presso il comune di Marigliano (...) lo ebbi il preciso incarico di non fare uscire nelle elezioni Francesco Montagna e di favorire la candidatura di Giacomo Calabria (...) Avevo appena assunto il mio ufficio quando il prefetto di Caserta mi invitò a recarmi da lui per conferire, e recatomici, egli volle che io rimanessi con lui la sera a cena, perchè avremmo allora conferito sulle elezioni che dovevano aver luogo. E la sera trovai (insieme al candidato ministeriale e altri notabili) Peppuccio Romano, da Aversa, che purtroppo imperversò per lungo tempo nella provincia di Caserta, avendo a lui sottomesse tutte le camorre locali. Durante la cena fu stabilito il piano di battaglia (...) lo fui messo agli ordini del Romano, ma dichiarai sin da quella sera che, pur mettendo ogni mia attività per fiaccare, coi mezzi che la legge metteva a mia disposizione, il predominio del Montag-

(4) G. Mosca, Teorica dei governi e governo parlamentare, Milano 1968 (1. a ediz., 1884), pp. 192-194.

(5) G. Salvemini, // ministro della malavita, Milano $1962\left(1 .^{\circ}\right.$ ediz.), p. 138.

(6) AP - leg. XXIII - Disc. Vol. I, p. 5.359. 
na, io non garantivo altro che la sincerità del risultato delle urne, assicurando che avrei impedito con tutti i mezzi le consuete alterazioni e falsificazioni» (7).

Ora, al di là del dubbio ruolo di difensore della legalità e della sincerità elettorale in cui si presenta il prefetto Flores, la sua testimonianza mi sembra molto significativa, in particolare per l'aspetto relativo alla subordinazione dei funzionari governativi ai capicamorra, come Peppuccio Romano, che è il perfetto corrispondente di un cacique spagnolo, e per l'ammissione, come egli scrive, di «consuete alterazioni e falsificazioni». E altrettanto significativo mi sembra un altro documento, citato da Salvemini nel súb libro; la lettera inviata ad un candidato, in prossimità delle elezioni, da un sindaco di un comune pugliese, il quale gli scriveva: «ll prefetto Caracciolo è stato molto abile e mi ha coadiuvato molto bene specialmente nel lavoro di eliminazione. Però lo vedo un pò scrupoloso circa i mezzi da me proposti pel conseguimento del fine comune. Sarebbe opportuno, urgentissimo, lo scioglimento dell'amministrazione comunale di Canosa!... Infonderebbe addirittura paura, la vittoria sarebbe più che assicurata (...) Occorrono delegati energici e fedeli... Occorre rinnovare e subito il brigadiere dei carabinieri di Ruvo, e tante tante cose che il prefetto da solo può accordare» (8).

Come si vede si tratta di una realtà molto familiare, persino nella terminologia - scioglimento di consigli comunali, delegati, ecc. - a coloro che si occupano delle vicende elettorali spagnole dello stesso periodo. Tuttavia credo che per verificare fino in fondo questo parallelismo sia opportuna, per quanto possibile, una comparazione più approfondita, a partire da alcune considerazioni quantitative.

A questo proposito mi è sembrato significativo adottare, come misuratore dell'estensione delle adulterazioni elettorali, il numero e la qualità delle contestazioni per brogli che, nell'uno e nell'altro paese, pervengono agli organi incaricati di deliberare sulla validità di tali contestazioni. Posto che tale comparazione può risultare tanto più indicativa quanto più sincronica, mi è parso meno dispersivo e anche più denso di significato, concentrare il confronto in un arco di tempo in cui i due paesi presentassero sistemi elettorali più simili.

In concreto però tali periodi sono due, ed entrambi piuttosto brevi: il primo che comprende le elezioni spagnole del 1879 e del 1881, e quelle italiane del 1880 , in cui entrambi i paesi hanno un sistema elettorale fondamentalmente censitario e a collegio uninominale; il secondo, ancora più breve, che comprende le elezioni italiane del 1913 e quelle spagnole del 1914, in cui i due

(7) F. Cordova, «Alcuni ricordi inediti d'un prefetto dell'età liberale», in Storia contemporanea, n. 2, 1974.

(8) G. Salvemini, // ministro..., cit. pp. 129-30. 
paesi hanno un sistema a suffragio universale e a collegio uninominale -anche se in Spagna i più importanti centri urbani hanno lo scrutinio di lista con voto plurimo limitato-, mentre in Italia nelle successive elezioni del dopoguerra si introdurrà il sistema proporzionale e a scrutinio di lista.

Ora, a parte la maggiore difficoltà di reperire i dati necessari alla comparazione per il primo periodo, mi è sembrato assai più significativo concentrare l'attenzione sul secondo, sia perchè in questo, per quanto riguarda la Spagna, il sistema della Restaurazione, ancorchè sia entrato in crisi, è già ampiamente collaudato e funziona a pieno regime, sia perchè, per quanto riguarda I'Italia, il perio do giolittiano, benchè, come si è visto, non sia il primo in cuisi verifica l'intervento governativo nelle elezioni, è certamente quello che gode di peggior fama sotto il profilo delle pratiche truffaldine. Per entrambi del resto, il suffragio universale amplifica le necessità di intervento governativo; per cui è ipotizzabile che, soprattutto in Italia, siano queste le elezioni in cui si registrano il massimo di brogli. Infine, essendo troppo breve il periodo del comune suffragio universale, ho ritenuto che, nonostante la diversità di sistemi elettorali, fosse ugualmente indicativa l'analisi delle contestazioni per frodi elettorali nelle due elezioni precedenti, che peraltro, per quanto riguarda l'Italia, costituiscono tutte quelle celebrate nella cosiddetta età giolittiana.

Fatte tutte queste premesse, se ora si osserva la tabella II in cui sono sintetizzati i dati di questa comparazione, vi sono delle risultanze che appaiono immediatamente evidenti. La prima è che le elezioni contestate in Italia per brogli in questo periodo sono molto poche, ed è una constatazione tanto più sorprendente se si considera lo scandalo da esse suscitato nel paese e la sinistra fama che gettarono sull'intero periodo giolittiano. La seconda evidente risultanza, strettamente correlata alla prima, è che quelle elezioni gravemente inquinate furono in Italia in numero notevolmente inferiore a quelle spagnole; in termini assoluti, e tanto più in proporzione al numero dei mandati parlamentari. La terza constatazione, più scontata, è che in Italia le elezioni invalidabili per brogli erano di più nel Mezzogiorno che nel resto del paese, benchè il risultato del 1909 non presenti differenze molto marcate.

Prima di passare a trarre delle indicazioni da queste risultanze, vorrei fare alcune considerazioni a proposito della attendibilità della fonte da cui si ricava per l'Italia quel numero sorprendentemente piccolo di elezioni inquinate da brogli.

La fonte è costituita dagli atti della Giunta parlamentare per la verifica dei poteri, detta comunemente Giunta delle elezioni, a cui era appunto demandato il compito di giudicare della validità dei ricorsi relativi alla regolarità delle elezioni. Ora, le Giunte che si susseguirono nelle varie legislature non furono esenti da alcuni comportamenti partigiani, non solo nei loro verdetti, ma a vol- 
te anche nel non accettare come contestate e quindi da sottoporre al suo giudizio, alcune elezioni sulle quali tuttavia erano stati presentati ricorsi per irregolarità (9). Nondimeno si tratta di episodi relativamente esigui, che non mancarono del resto di suscitare clamori, e che non possono alterare sostanzialmente i dati che da quella fonte si ricavano.

La garanzia fondamentale della sua attendibilità sta infatti principalmente nella composizione delle Giunte e nello strumento, di cui disponevano le minoranze che ne facevano parte, di presentare alla Camera, cui spettava il giudizio definitivo, una relazione avversa alle delibere della maggioranza. Per quanto riguarda il primo punto, vediamo infatti che nelle tre legislature prese in esame non solo i fedelissimi giolittiani sono poco rappresentati nelle Giunte, ma che di esse fa parte una crescente e qualificatissima rappresentanza socialista. In essa si contano personaggi come Andrea Costa, padre fondatore del partito, Camillo Prampolini, Giacomo Ferri e lo stesso Filippo Turati.

Ora, sappiamo che Giolitti, soprattutto nel primo perio do della sua egemonia politica stabilí buoni rapporti con i socialisti e certo questo fatto si potè riflettere sulla loro intransigenza anche in materia elettorale (10). Tuttavia non bisogna esagerare questo atteggiamento di eventuale condiscendenza o complicità, perchè laddove si presentano irregolarità gravi essi danno battaglia in Giunta e fuori. Il caso di Gioia del Colle denunciato da Salvemini nel suo libro, ma che ha un antefatto nella Giunta delle elezioni e sulla stampa, è emblematico, perchè il candidato danneggiato dai brogli e le violenze dei giolittiani non era un socialista ma un grande proprietario conservatore.

Quando dunque la minoranza - che, oltre che da socialisti, poteva essere costituita anche da repubblicani, radicali, soniniani, ecc.-, riteneva che la maggioranza convalidasse un'elezione contro l'evidenza di brogli, si dissociava

(9) In realtà il fenomeno si verificò in forma grave solo alle elezioni del 1913, poichè, come ebbe a dichiarare Filippo Turati, durante la discussione nell'aula parlamentare a proposito dell'elezione del collegio di Roma $1 .^{\circ}$, che, nonostante i ricorsi e le proteste, era stata dichiarata «non contestata» dalla Giunta delle elezioni, si trattava di una «enormità non mai verificatasi nella Camera italiana». E più appresso spiegava: «In passato avveniva che per certe proteste di poco conto, si discutesse rapidamente e magari si passasse oltre; era una irregolarità formale, ma sostanzialmente giustificata dalla necessità di non moltiplicare all'eccesso le contestazioni, e dal consenso unanime o quasi dei commissari. Oggi si è arrivati all'estrema conseguenza, all'assurdo, cioè, di abolire, anche nei casi più gravi, le contestazioni» (AP - Leg. XXIV - Disc., vol. I, p. 361).

(10) Nel quadro di queste possibili intese tra ministeriali e socialisti si registra, ad esempio, un episodio poco encomiabile nella limpida condotta politica di Filippo Turati, il quale, nel 1909 -anno in cui peraltro in Giunta si accese la disputa sulla famosa elezione di Gioia del Colle - fu relatore di maggioranza contro l'annullamento di un'elezione, in cui il candidato ricorrente, il cattolico Giovanni Andrea Ronchi, era risultato perdente perchè i voti espressi per «Giovanni Ronchi» e "Giovanni Andrea Ronchi» erano stati computati come se fossero da attribuire a due candidati diversi (Cfr. AP - Leg. XXIII, Doc., vol. I, VIII, n. 3, p. 1 e ss). La Camera poi, sulla base di quanto proposto dalla Giunta, respinse il ricorso convalidando l'elezione. 
da quella convalida, non solo votando contro di essa, ma presentando le sue controdeduzioni in una relazione di minoranza, che, come quella della maggioranza, era oggetto di discussione da parte della Camera nella seduta in cui si sarebbe deliberato in via definitiva (11). Ora, a conferma di una sostanziale correttezza dell'operato della Giunta, e quindi della attendibilità dei dati che da essa provengono, c'è, come si può vedere nella tabella, un numero piuttosto piccolo di relazioni di minoranza, che peraltro furono sempre precedute da discussioni molto accese, e d'altro canto un numero piuttosto alto di annullazioni o rettifiche delle elezioni contestate - si noti che nel 1909 riguardano quasi tutte le elezioni sut judice - che fa risaltare l'enorme sproporzione che invece si registra nel caso della Spagna.

In definitiva, al di là di ogni altra considerazione, credo che, a meno che non vi fosse a monte della Giunta delle elezioni un filtro alle denunce di brogli e coazioni - di cui però non ho trovato alcun riscontro-, il numero delle elezioni invalidabili per brogli, e quindi il volume dei brogli commessi, fosse quello che si ricava dagli atti della Giunta stessa. D'altro canto, una conferma della esiguità di quel numero ci viene anche da una testimonianza insospettabile, quella dello stesso Gaetano Salvemini, il quale in un articolo su L'Unità, in prospettiva delle elezioni del 1913, scriveva: «(ll governo) concentra le forze su quei soli punti, in cui bisogna far vincere ad ogni costo un servitore del ministero, e sbarazzarsi di un avversario. Non sono mai più di un paio di dozzine di collegi in tutto il Mezzogiorno» (12).

Potremmo fare molte altre considerazioni a proposito dei dati a confronto che appaiono in quella tabella. La fondamentale delle quali è che quella grande sproporzione che vi risulta, tra i brogli commessi in Spagna e in Italia, potrebbe essere fortemente attenuata se si potesse provare che in Spagna, il solo e più infondato ricorso contro un'elezione, bastava perchè fosse sottoposta a giudizio, e che perciò poi l'organo giudicante, nella massima parte dei casi, ne attestava l'infondatezza. Ma a parte che in linea di massima si può affermare che quell'ipotesi non corrisponde affatto alla realtà, la sproporzione è tale che comunque resterebbe tra i due paesi una differenza molto grande.

Questo significa che in Italia, e in particolare nel Mezzogiorno, le elezioni erano più corrette che in Spagna? Non credo. Ciò che cambia è il tipo di deviazioni, o meglio l'incidenza di un diverso tipo di deviazioni dal modello di un corretto funzionamento delle elezioni nel sistema liberaldemocratico.

(11) La Camera quasi sempre approvava le proposte della Giunta o della sua maggioranza. I casi di proposte della Giunta non approvate dalla Camera furono 4 nel 1904, 3 nel 1909 e 3 nel 1913.

(12) L'Unitá, 8.VIII.1913. 
In cosa consistesse questo diverso tipo di deviazioni lo descriveva, nel 1880 , il deputato Pietro La Cava nel suo libro Sulla Riforma della legge elettorale, nel quale scriveva: «In generale nei nostri collegi le elezioni si fanno per relazioni più personali che politiche. In ogni paese o borgata vi sono due o tre capiparte o grandi elettori: guadagnati questi, l'elezione è assicurata (...) II grande elettore deve mantenere la sua influenza a spese del deputato pel quale l'ha adoperata nel giorno dell'elezione. Ed in che modo? In un governo accentrato come il nostro, nel quale per nove decimi dipende la trattazione degli affari dagli agenti del potere esecutivo, la risposta è troppo facile ed evidente. Qui è il Sindaco o vice-pretore da nominare o destituire, là un Consiglio comunale o provinciale da sciogliere, colà un istituto pio da riformare; qui è un magistrato da traslocare, là un altro funzionario da promuovere, colà un altro agente da collocare». La Cava proseguiva con un lungo elenco di favori leciti e meno leciti, per poi concludere: «E cosí per una catena di interessi infiniti e indefiniti, il rappresentante della nazione addiviene sollecitatore d'affari, ed il potere legislativo entra ad ampie vele nell'ambiente del potere esecutivo. Ed alla sua volta il potere esecutivo ne trae profitto nelle grandi occasioni facendo giuocare questi interessi nei giorni critici delle votazioni, pei quali interessi non sono rari i casi di risoluzioni prese o sospese in contraddizione di leggi e regolamenti» (13).

É una realtà quella rappresentata dal La Cava _il quale come direttore generale del ministro degli Interni Nicotera aveva fatto una larga esperienza di queste pratiche durante le elezioni del 1876 - che sembra ricalcare in larga misura il fenomeno del caciquismo spagnolo nella sua dimensione nazionale. Anche la dipendenza dell'esecutivo dai deputati, a loro volta espressione delle clientele locali, impone in Italia come in Spagna l'ingerenza governativa nelle elezioni.

Questa relazione di necessità è descritta con molta lucidità ancora dal Mosca il quale scriveva: «Là dove i deputati, creando il ministero, si riservano sempre il diritto di cambiarlo a lor voglia, il ministero reagendo, alla sua volta crea, per mezzo dei prefetti, una quantità di deputati; i quali naturalmente sono interessati a sostenerlo sempre ed a non farlo mai mutare». E a proposito dei sistemi adoperati dai prefetti per raggiungere quello scopo, più avanti aggiungeva: «In quanto ai mezzi con cui i prefetti esercitano la loro influenza, essi sono vari. Prima di tutto hanno un certo numero di elettori, impiegati, persone dipendenti, ecc., dei cui voti sono sicuri, ma questi non sogliono essere molti; poi ricorrono all'espediente di venire a trattative con gli elementi che nei singoli paesi si impongono, cioè con le società politiche e specialmente con i grandi elettori, ed a questi fanno dare direttamente dal governo quei

(13) Riportato in: M. Minghetti, Scritti politici, Roma 1986, n. 1, pp. 612-13. 
medesimi favori, che essi ordinariamente ottengono per mezzo dei loro deputati. L'amministrazione provinciale e comunale offre pure loro molte risorse. E' cosí che, quando i prefetti hanno del tatto e della scaltrezza, riescono spessissimo a riunire un numero di voti preponderante sui candidati raccomandati dal ministero" (14).

Ecco dunque qui delineata la somiglianza e al tempo stesso quella che è a mio parere la chiave di una sostanziale differenza tra i due paesi.

La somiglianza consiste nel comune tessuto clientelare delle due società -ma per quanto riguarda l'Italia, è importante sottolineare che ciò riguarda, soprattutto il Meridione- che snatura il sistema politico liberaldemocratico. Ma in Italia l'obiettivo del prefetto era, come scrive il Mosca, «riunire un numero di voti preponderante sui candidati raccomandati dal ministero»; vale a dire che egli, come i grandi elettori, nella massima parte dei casi, non impiegava frodi, ma si avvaleva di tutti i metodi clientelari che erano alla sua portata, per ottenere per il candidato governativo i voti degli elettori. II fondamentale fattore delle elezioni nell'Italia meridionale era cioè il tipico scambio clientelare: favori in cambio di voti (15).

\section{IL PESO DEI BROGLI NELLE ELEZIONI SPAGNOLE}

In Spagna non è cosí. Non perchè quel tipo di scambio non esistesse o fosse del tutto trascurabile. Ma perchè in definitiva il fattore decisivo delle elezioni, era prevalentemente, e direi quasi necessariamente, il broglio.

In mancanza di altri supporti quantitativi a questa indicazione, oltre a quello già fornito relativo alle elezioni impugnate per frode, vorrei aggiungere alcune osservazioni che, benchè non abbiano lo stesso peso dimostrativo, mi sembrano ugualmente molto significative.

(14) G. Mosca, Teorica..., cit. pp. 248-49.

(15) II fatto che questo fosse il meccanismo essenziale delle elezioni piuttosto che i brogli ci è attestato anche da quanto scriveva, a proposito delle elezioni del 1886, l'anarchico Francesco Saverio Merlino, ovviamente assai poco indulgente nei confronti dei poteri pubblici. «ll governo - affermava in un suo pamphlet pubblicato a Parigi nel 1890- studiò i bisogni delle popolazioni, o più esattamente, della maggioranza elettorale e si affrettò a soddisfarli. II ministro dell'Interno accordava sussidi agli asili, permessi di porto d'armi, riscatti di multe, scuole, ecc.; quello della Guerra concedeva ad un comune una caserma, all'altro la guarnigione; il ministro di Grazia e Giustizia firmava grazie, quello dei Lavori pubblici accordava strade da gran tempo richieste dai comuni, permessi di piantare risaie a dispetto dei pareri del consi gliere sanitario, salvo ad annullare più tardi la sua concessione: e in tutti questi casi il Ministro, con un bibliettino di suo pugno e il giornale ufficiale locale avevano cura di far conoscere alle popolazioni, ch'esse dovevano la loro fortuna ai buoni uffici del candidato ministeriale. II deputato Cavallotti aveva motivo di domandare ai ministri: «Avete chiamato gli elettori ad approvare la vostra politica, oppure a farvi ringraziare per i vostri favori?» (F. S. Merlino, Questa è l'Italia, Milano 1953 (París, 1890), pp. 169-160. 
Nel 1913, il socialista Camillo Prampolini relatore della Giunta delle elezioni a proposito dell'elezione del collegio di Melfi, di cui proponeva l'invalidazione, commentava il fatto che nella sezione di Rionero il candidato vincitore avesse ottenuto la quasi totalità dei suffragi espressi, affermando: «Non ci consta che vi siano altri esempi di una cosi imponente e quasi assoluta unanimità» (16). $E$ in effetti si trattava di un caso rarissimo nelle elezioni italiane, che non mancava di suscitare, come avvenne anche per le elezioni di Gioia del Colle, grande scandalo. Ora chiunque abbia anche solo una minima esperienza delle elezioni spagnole sa quanto invece fosse frequente un caso del genere: «caso inverosimile - come lo definiva una sentenza del Tribunal Supremo del 1919«ma non certo raro e singolare, perchè è fin troppo abituale e corrente questo raggiro tra i professionisti (dei brogli elettorali)» (17).

Questo en plein di voti in poche sezioni, era spesso sufficente ad attribuire la vittoria elettorale. Esso consentiva quindi di conseguire il risulato voluto con un intervento concentrato su pochi punti, ed era spesso il modo attraverso il quale, con il voto unanime di alcune poche sezioni rurali, si sovvertiva il voto dei centri urbani. E' evidente che per realizzare questo pieno di voti era necessario avere il controllo assoluto del seggio, e sappiano anche che una condizione essenziale per averlo, era avere il controllo del municipio, che d'altronde consentiva anche altre manipolazioni elettorali. Un'ulteriore significativa indicazione di quale importanza rivestisse la frode nel sistema elettorale spagnolo è allora costituita dalla grande quantità di cambiamenti di sindaci e interi consigli comunali che precedevano le elezioni.

Non sono riuscito a trovare rilevazioni statistiche che potessero indicare la dimensione esatta di questo fenomeno. Solo per le elezioni del 1910 ho trovato un rapporto redatto ad uso del ministro degli Interni, da cui risulta che, nella prospettiva della prova elettorale, erano stati sospesi nei mesi precedenti 162 sindaci, 732 consiglieri comunali e 35 interi consigli comunali (18). Non si tratta di cifre enormi, ci sono però alcuni indizi, come vedremo, che lasciano pensare che si tratti di dati incompleti. Ma, oltre alle sospensioni, bisogna aggiungere il gran numero di sindaci di nomina regia che venivano sostituiti, e soprattutto i moltissimi alcaldes e ayuntamientos che spontaneamente e, più spesso, in seguito a pressioni e ricatti, si dimettevano.

Se si guarda la corrispondenza dei governatori in periodo preelettorale, si vede subito quanta della loro attività fosse rivolta a conseguire questi cambiamenti nei consigli comunali, e quanto decisivo lo ritenessero per la vittoria

(16) AP - Leg. XXIV, Doc., Vol. 1, V, n. ${ }^{\circ} 59$, p. 2.

(17) DC, 1.7.1919, ap. 8 al n. 4, p. 3.

(18) AHN, Gobernación serie A, fascicolo 10 A, busta 2. 
dei candidati del governo. Gli esempi potrebbero essere infiniti. Nei telegram$\mathrm{mi}$ tra governatori e ministro degli Interni sono frequentissime le affermazioni come questa che troviamo in un dispaccio inviato dal governatore di Cuenca in occasione delle elezioni del 1910, in cui si dice: «E'urgentissimo che il candidato del collegio di Cañete Sr. Rengifo si presenti quanto prima perchè dopo che abbiamo sospeso 18 Consigli comunali, si dà il caso che il suddetto candidato non ha escrito neppure una lettera agli elettori» (19).

Ho scelto questo tra i molti esempi che avrei potuto portare, da una parte perchè mostra come alcuni candidati, abituati al fatto che il governo si occupava di tutto, non si disturbavano nemmeno a scrivere una lettera, dall'altra, perchè quei 18 ayuntamientos sospesi, che non risultano nel documento che prima ho citato, mostrano come questo fosse incompleto e che pertanto le cifre che esso indicava vadano certamente accresciute.

Molti altri esempi indicano come le pedine fondamentali nella scacchiera elettorale fossero molto di più gli ayuntamientos, con l'implicita possibilità che essi avevano di manipolare i risultati delle elezioni, che non gli elettori. Particolarmente espressivo è a questo proposito quello che in una lettera, citata da Tussell nel suo libro sul caciquismo in Andalusia, scriveva, in occasione delle elezioni del 1907, al ministro Juan de la Cierva, un candidato liberale, che però era suo amico personale: «lo ti chiedo in giustizia di ordinare al governatore di disporre sensa scuse nè pretesti la reintegrazione nei loro posti dei titolari dei Consigli comunali di (...) In tal modo equilibrerai un poco le condizioni di questa violentissima lotta. Avevo 18 consigli comunali; me ne hanno lasciati 2 ! Di questi 2, uno è insignificante; se me ne restituisci 7 i miei avversari restano con gli 8 ai quali con astuzie, minacce e tradimenti strapparono le dimissioni e ora non mi possono essere resi» (20).

Non credo sia necessario oltre sul peso dei brogli nelle elezioni spagnole insistere di questo periodo. Perché d'altra parte la necessità, la imprescindibilità del broglio per il perpetuarsi del sistema, era implicita nella regola stessa del turno, il cui sistematico funzionamento - sappiamo infatti con quanta regolarità abbia funzionato- non poteva basarsi che sulla frode. Ne discende quindi che pur configurandosi, tanto la spagnola che l'italiana, soprattutto nella sua area meridionale, come società di clientele, in Spagna il peso relativo dei brogli sull'esito elettorale era molto maggiore che in Italia. $O$, detto in altro modo, in Spagna, a differenza che in Italia, il sistema clientelare era incompatibile con la correttezza formale delle elezioni.

(19) AHN, Gobernación serie A, fascicolo 23 A, busta 10, tel. n. 641 del 14.4.1910.

(20) J. Tusell, Oligarquía y caciquismo en Andalucia (1890-1923), Barcelona 1976, p. 94. 


\section{Spagna e Italia: le ragioni della diversità}

Credo che questa sia la diversità sostanziale nel pratico funzionamento del sistema elettorale liberaldemocratico nei due paesi. Per quanto riguarda le ragioni di questa diversità posso solo abbozzare delle ipotesi, riferibili a loro volta ad alcune differenze del loro tessuto socio-politico.

A me sembra che una basilare differenza consista nel grado di coinvolgimento, di identificazione tra struttura clientelare della società e struttura politica. Per quanto riguarda la Spagna, Romero Maura nel suo noto articolo sul caciquismo (21) proponeva una visione di quel fenomeno in cui la struttura caciquil e quella politica erano fondamentalmente distinte. Cosí che la seconda era costretta a tollerare la prima, con la sua corruzione, perchè solo i caciques le potevano garantire, attraverso la manipolazione delle elezioni, la stabilità del sistema politico, che si fondava sulla regola del turno.

Credo che le cose non stessero cosí. Varela Ortega nel suo libro dà un'indicazione contraria molto chiara, che a mio parere ci può fornire una chiave di lettura più convincente. «l governi -scrive Varela- erano disposti a cedere parte del loro potere come amministratori ad uso ed abuso dell'organizzazione locale del partito (...) E questo non avveniva principalmente perchè, come a prima vista potrebbe sembrare, l'esecutivo avesse bisogno dei caciques e delle fazioni per fare le elezioni (visto che tanto gli uni che le altre controllavano un numero di distretti molto inferiore a quelli che il governo teneva sotto controllo dal ministero degli Interni) (...) Gli uomini della Restaurazione tollerarono e persino diedero un'organizzazione di alternanza al caciquismo esistente, perchè nessuno si vedesse escluso e pertanto posto nella necessità di ricorrere alla violenza per assicurare alla sua clientela l'uso di parte di strumenti e risorse dell'amministrazione» (22).

Ecco, questo è il significato profondo del sistema della Restaurazione. Un significato che peraltro è implicito nella regola stessa del turno. Essa è infatti incompatibile non solo con una competizione tra partiti di diversa ispirazione ideologica, ma anche con quella tra partiti di una stessa matrice ideologica che si contrappongono per la sola diversità di programmi di governo. II senso di quel sistema era dunque quello dell'uso alternato delle risorse messe a disposizione dal potere statale, cioè della spartizione delle spoglie.

Per tanto la rete delle clientele quasi non conosceva distinzione tra amministratori, caciques e politici, e come è noto, non si limitava all'ambito locale;

(21) Cfr. J. Romero Maura, «El caciquismo: tentativa de conceptualización, in Revista de Occidente, n. 127, 1973.

(22) J. Varela Ortega, Los amigos políticos (partidos, elecciones y caciquismo en la Restauración (1875-1900), Madrid, 1977, pp. 440-41. 
attraverso i caciques di scala provinciale e regionale, essa coinvolgeva i vertici politici e burocratici di livello nazionale. Vi era cioè intima solidarietà tra centro e periferia, e tra i ruoli, politico e burocratico -spesso intercambiabilinell'uso del potere statale, se non sempre in termini di aperta corruzione, certo al fine precipuo di beneficiare una parte limitata della società.

La limitatezza dei beneficiari dipendeva dalla limitatezza delle risorse disponibili, la quale peraltro implicava la necessità della frode elettorale. Da una parte infatti, è proprio quella limitatezza a rendere impossibile su grande scala, quel meccanismo che abbiamo visto essere caratteristico delle elezioni italiane, lo scambio cioè di favori e benefici per voti. Nella Spagna di allora quei benefici erano infatti la meta della competizione elettorale, e non potevano pertanto essere sprecati per conseguire un potere politico svuotato in gran parte di risorse. D'altro canto, la limitatezza delle risorse imponeva, per la stabilità del sistema, anche l'alternanza nel loro sfruttamento. Di qui la necessità del turno, e ancora della frode, per vincere le resistenze, sia delle clientele che non accettavano l'avvicendamento sia, in minor misura, di quella parte dell'elettorato che era costantemente esclusa dalla spartizione.

Per quanto riguarda l'Italia, si potrebbe forse ipotizzare che se il paese fosse stato tutto come il Mezzogiorno, la sua somiglianza con la Spagna sarebbe stata assai più accentuata. Indubbiamente nel determinare la diversità del caso italiano ha influito in modo decisivo il fatto che in Italia coesistevano due società, la settentrionale e la meridionale, di dimensioni pressocchè uguali, che presentavano delle differenze economico-sociali molto marcate (in Spagna, pur esistendo differenze regionali, erano meno rilevanti, e suprattutto c'era un grande squilibrio di dimensioni tra aree dinamiche e aree arretrate, nel senso che le prime erano troppo circoscritte per poter condizionare le seconde).

Questo fatto, di un Nord molto più avanzato e prospero, principale portatore ed esecutore del movimento unitario, ha permesso che i governi italiani potessero perseguire e in parte realizzare delle politiche che trascendevano il sistema clientelare. E che anzi, a volte potessero valersi del sistema clientelare per realizzare delle politiche progressive, anche se pagando un prezzo la cui accettabilità è ancora oggi oggetto di discussione. Ad esempio, Giolitti favoriva le clientele meridionali anche allo scopo - che Salvemini ben conosceva senza per questo assolverlo -di procurarsi la base parlamentare indispensabile alla sua politica di sviluppo del riformismo sociale della classe operaia del Nord.

La maggiore ricchezza del Settentrione forniva inoltre ai governi italiani quel patrimonio di risorse da spendere per poter acquisire il voto del Meridione. Ed é proprio questo che mancava ai governanti spagnoli. Essi non avevano molte possibilità di costruire strade, ponti, scuole, caserme, ecc.; o di favo- 
rite crediti, concedere sconti sulle imposte, o creare molti nuovi impieghi. Perciò i Silvela, i Canalejas, i Maura, ecc., in molti casi al di là della loro volontà soggettiva, erano irrimediabilmente condannati dalla scarsità di risorse a compiere soltanto la funzione di grandi sacerdoti della regola del turno.

Infine vorrei osservare che anche il maggior dinamismo economico impresso dal Settentrione in qualche misura a tutto il paese, contribuí in Italia ad arginare, anche se certo in modo insufficente, l'assalto delle clientele allo Stato. Giustino Fortunato, uno dei nostri grandi meridionalisti, poneva l'accento sullo stretto rapporto tra sviluppo delle relazioni clientelari e carenza delle attività imprenditoriali, quando scriveva: «Finchè mondo sarà mondo, non cesseranno mai i rapporti personali fra deputati ed elettori, saranno anzi eccessivi in Italia, finchè avremo tanta e così minuta borghesia non industriale nè commerciale, costretta a non vedere altra salute se non nella impiegomania» (23).

Se questo era vero in Italia, tanto più lo era in Spagna, dove sviluppo dell'agricoltura, commercializzazione dei suoi prodotti e industrializzazione erano senza dubbio più indietro. Perciò quell'uso del potere statale in funzione di una ascesa economica e sociale da parte di gruppi limitati, che caratterizza il sistema della Restaurazione, più che corrispondere a una vocazione ostinatamente parassitaria diffusa nella società spagnola, credo invece che spesso costituisse l'unica opportunità di mobilità sociale verso l'alto, in mancanza di altre consistenti occasioni produttive su cui edificare quell'ascesa. Non a caso, quando con il progredire dell'industrializzazione quelle occasioni si sono presentate con maggiore frequenza, l'uso clientelare dello Stato ha perduto consistenza, almeno in comparazione con il periodo storico di cui ci stiamo occupando.

(23) G. Fortunato, // Mezzogiorno e lo Stato italiano Firenze 1973 (1. ${ }^{\circ}$ ediz. 1926) I, pp. 92-3. 


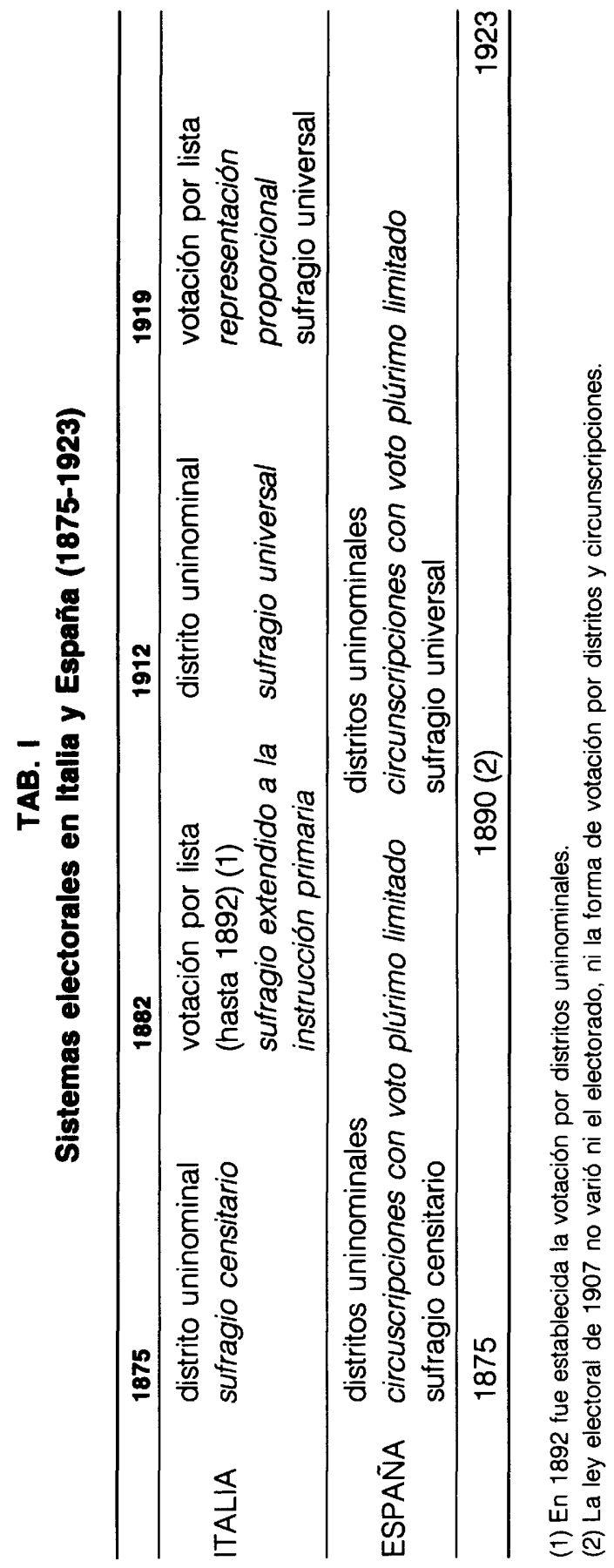




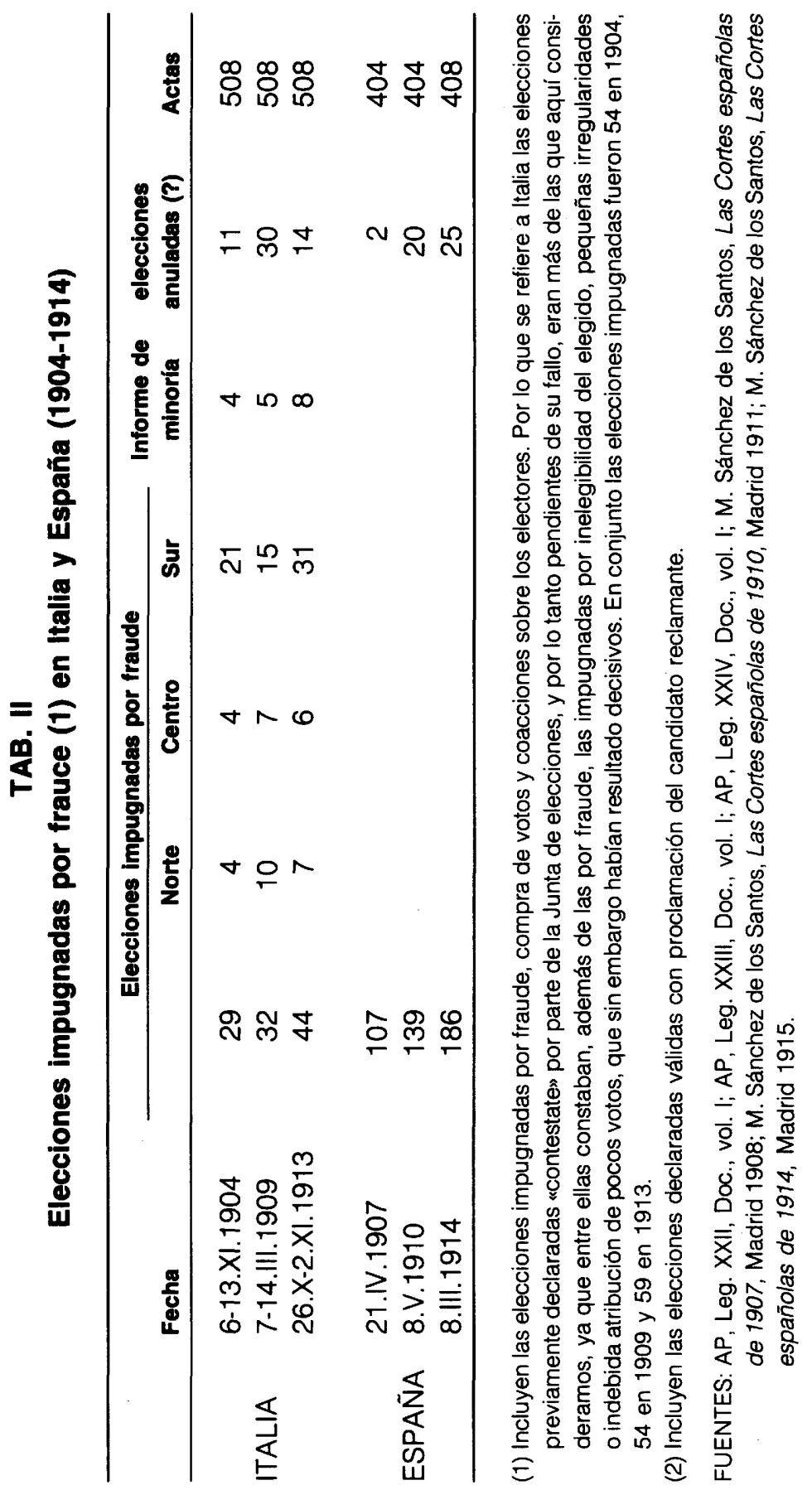

